\title{
Stellar populations in the CFHTLS ${ }^{\star}$
}

\section{New constraints on the IMF at low mass}

\author{
M. Schultheis ${ }^{1,2}$, A. C. Robin ${ }^{1}$, C. Reylé ${ }^{1}$, H. J. McCracken ${ }^{2,3}$, E. Bertin ${ }^{2,3}$, Y. Mellier ${ }^{2,3}$, and O. Le Fèvre ${ }^{4}$ \\ 1 CNRS UMR6091, Observatoire de Besançon, BP 1615, 25010 Besançon Cedex, France \\ e-mail: mathias@obs-besancon.fr \\ 2 CNRS UMR 7095, Institut d'Astrophysique de Paris, 98bis Bld. Arago, 75014 Paris, France \\ 3 Observatoire de Paris, LERMA, 61 avenue de l'Observatoire, 75014 Paris, France \\ ${ }^{4}$ Laboratoire d'Astrophysique de Marseille, OAMP, Université de Provence, UMR 6110, Traverse du Siphon-Les trois Lucs, 1302 Marseille, \\ France
}

Received 6 May 2005 / Accepted 12 September 2005

\section{ABSTRACT}

We present a stellar populations analysis of the first release of the CFHTLS (Canada-France-Hawai Telescope Legacy Survey) data. A detailed comparison between the Besançon model of the Galaxy and the first data release of the CFHTLS-Deep survey is performed by implementing the MEGACAM photometric system in this model using stellar atmosphere model libraries. The reliability of the theoretical libraries to reproduce the observed colours in the MEGACAM system is investigated. The locations of various stellar species like subdwarfs, white dwarfs, late-type and brown dwarfs, binary systems are identified. The contamination of the stellar sample by quasars and compact galaxies is quantified using spectroscopic data from the VIMOS-VLT Deep Survey (VVDS) as a function of $i^{\prime}$ magnitude and $r^{\prime}-i^{\prime}$ colour. A comparison between simulated counts using the standard IMF at low masses show that the number of very low mass dwarfs may have been underestimated in previous studies. These observations favour a power law IMF following $\mathrm{d}(n) / \mathrm{d} m \propto m^{-\alpha}$ with $\alpha=2.5$ for $m<0.25 M_{\odot}$ or $\alpha=3.0$ for $m<0.2 M_{\odot}$ for single stars. The resulting LF is in agreement with the local LF as measured from the 5 or 25 pc samples. It is in strong disagreement with the Zheng et al. (2001) LF measured from deep HST data. We show that this discrepancy can be understood as an indication of a different IMF at low masses at early epochs of the Galaxy compared to the local thin disc IMF.

Key words. Galaxy: stellar content - stars: luminosity function, mass function - stars: white dwarfs - binaries: general - stars: atmospheres stars: low mass, brown dwarfs

\section{Introduction}

The CFHTLS (Canada-France Hawaii Telescope Legacy Survey) is a five year large observing program at the $\mathrm{CFH}$ Telescope, using the wide field prime focus MegaPrime equipped with MEGACAM, a 36 CCD mosaic camera. Together with its small pixel scale of 0.185 arcsec and the large number of nights dedicated to the survey (around 500 nights over five years), the CFHTLS goes deeper and has a better image quality than the Sloan Digitized Sky Survey but on a much smaller area of the sky. Hence it probes a different volume of

* Based on observations obtained with MegaPrime/MEGACAM, a joint project of CFHT and CEA/DAPNIA, at the Canada-FranceHawaii Telescope (CFHT) which is operated by the National Research Council (NRC) of Canada, the Institut National des Science de l'Univers of the Centre National de la Recherche Scientifique (CNRS) of France, and the University of Hawaii. This work is based in part on data products produced at TERAPIX and the Canadian Astronomy Data Centre as part of the Canada-France-Hawaii Telescope Legacy Survey, a collaborative project of NRC and CNRS. the Universe. The scientific goals from CFHTLS cover a wide range of scales: from the solar system (systematic search of trans-Neptunian objects), stellar populations and galactic structure, up to the distant universe, constraining the geometry of the universe (SNIa and cosmic shear), dark matter properties, quasars, clusters of galaxies, properties of galaxy clustering and galaxy evolution at high redshift, and probing the relation between dark and luminous matter.

We plan a series of papers dedicated to analysing stellar populations in the different surveys components of the CFHTLS. In this paper we analyse a subset of the first data release of the CFHTLS, investigating the objects classified as stellar in the catalogues. We examine the photometric quality of the three fields of the Deep Survey, D1, D2 and D3, determine the stellar populations and the contamination by compact galaxies and quasars. Using the Besançon model of stellar population synthesis (Robin et al. 2003) together with stellar atmosphere models Basel3.1 (Lejeune et al. 1997; Westera et al. 2002) and NextGen (Allard et al. 1997), we are able to produce 
Table 1. Location, median seeing and field of view (in sq. degree) of the 3 CFHTLS fields studied here.

\begin{tabular}{ccccccc}
\hline \hline Field & $\alpha(J 2000)$ & $\delta(J 2000)$ & $l(\mathrm{deg})$ & $b(\mathrm{deg})$ & seeing in $i^{\prime}$ & FOV \\
\hline D1 & $02 \mathrm{~h} 26 \mathrm{~m} 00 \mathrm{~s}$ & $-04 \mathrm{~d} 30 \mathrm{~m} 00 \mathrm{~s}$ & 172.0 & -58.0 & 0.88 & 0.80 \\
D2 & $10 \mathrm{~h} 00 \mathrm{~m} 29 \mathrm{~s}$ & $+02 \mathrm{~d} 12 \mathrm{~m} 21 \mathrm{~s}$ & 236.8 & 42.1 & 0.95 & 0.69 \\
D3 & $14 \mathrm{~h} 19 \mathrm{~m} 28 \mathrm{~s}$ & $+52 \mathrm{~d} 40 \mathrm{~m} 41 \mathrm{~s}$ & 96.2 & 59.6 & 0.92 & 0.77 \\
\hline
\end{tabular}

synthetic star counts, colour-colour and colour-magnitude diagrams in the MEGACAM filter system. We determine the location of white dwarfs, brown dwarfs and binaries in the different colour-colour diagrams. We emphasize in this paper the study of the luminosity function at low masses of the disc population. A comparison between star counts of the M dwarf populations in the three CFHTLS Deep fields and model predictions provide new constraints on the slope of the IMF of low-mass stars, a parameter which is still under debate.

\section{The CFHTLS fields}

The CFHTLS survey consists of three different surveys:

- The Deep, a survey comprising four fields (named D1, D2, D3, D4) of 1 square degree each, in five filters $u^{*}, g^{\prime}, r^{\prime}, i^{\prime}$ and $z^{\prime}$ reaching $r^{\prime}$ up to $28^{\mathrm{m}}$, observed every 3-4 days,

- the Wide, a survey of 3 patches (W1, W2, W3) each around $7 \mathrm{deg}^{2}$ in five bands, with limiting magnitudes $r^{\prime}$ up to $25^{\mathrm{m}}$, observed in two epochs separated by 3 years,

- the Very Wide, a survey of $1300 \mathrm{deg}^{2}$ along the ecliptic on 5-6 epochs, dedicated to the detection of fast moving solar system objects and stellar populations.

We refer the reader to the corresponding web pages (http://www.cfht.hawaii.edu/Science/CFHLS/) for a detailed description of the survey components. The observations are carried out at the CFHT using the MEGACAM camera, which consists of a mosaic of $36 \mathrm{CCDs}$, each of them $2048 \times 4612$ pixels large. The pixel scale is $0.185^{\prime \prime}$ which gives a total field of view of $0.96 \times 0.94 \mathrm{deg}^{2}$. The seeing of the observations used to construct the stacks is better than $1.1^{\prime \prime}$, which guarantees high quality data for the three different surveys.

We present in this paper an analysis of three fields of the "Deep" Survey, D1, D2 and D3 of the CFHTLS release T0001. These data are stacks of many images. Field coordinates and the median seeing in $i^{\prime}$ in each field are given in Table 1 .

\section{Data reduction}

The stacks and catalogues used in this paper were released as part of the TERAPIX T0001 public release. A brief outline of how these stacks were prepared is as follows.

CFHTLS observations are carried out with MEGACAM in queue survey mode. For release T0001, only observations from June, 1st 2003 to July, 22, 2004 were used. Pre-reductions were carried out at the CFHT using the ELIXIR ${ }^{1}$ pre-reduction system at CFHT and then these pre-reduced images were shipped to TERAPIX via the Canadian Astronomy Data Centre,

\footnotetext{
${ }^{1}$ http://www.cfht.hawaii.edu/Instruments/Elixir/
}

in Victoria, Canada. These pre-reduced images were then injected into the TERAPIX pipeline for inspection and quality control purposes. The TERAPIX tool QualityFITS was used to inspect and grade each image, and also to produce weightmaps derived from the CFHT-provided master flats using the WeightWatcher tool. The global astrometric and photometric solutions were computed using the WIFIX package, an earlier generation of the TERAPIX astrometric software, as the production astrometric software package was still in testing phase at the time of the T0001 release. For inclusion in the stacks, images must have a seeing better than $1.1^{\prime \prime}\left(1.3^{\prime \prime}\right.$ in $\left.u^{*}\right)$ and airmass less than 1.5. From this point on, for the image reductions, we followed essentially the same procedure as outlined in McCracken et al. (2003), and we refer to the interested reader to this paper for more details. The two significant differences are firstly that we use weight maps computed from the image flatfields themselves and secondly we use the USNO-B as the astrometric reference catalogue (which increases the robustness of the overall astrometric solution with respect to the solutions utilising the USNO-A). Full details of the properties of the final stacks, including depth in each filter and the accuracy of the final astrometric solution can be found on the TERAPIX web pages ${ }^{2,3}$. The internal accuracy of the astrometric solution (band-to-band) is better than one pixel rms over the entire MEGACAM field, whereas the external astrometric solution is around $\sim 0.25^{\prime \prime}$ rms.

Photometric calibrations for each pre-reduced image is provided by the ELIXIR pipeline. ELIXIR also applies a CCDto-CCD flux scaling derived from repeated observations of dense stellar fields which are shifted many times around the MEGACAM field of view (providing magnitude measurements of the same star on different CCDs). This procedure is necessary to correctly account for the "scattered light" effect and ensures that the flux of any given object is independent of the position on the mosaic. The residual ccd-to-ccd magnitude error following this procedure is around $\sim 3 \%$. In constructing the final stacks, we compare the magnitudes of objects in overlapping pointings and in each band the photometric exposures are indentified as those in which the objects have the highest flux: other images are scaled to these observations. Based on an examination of galaxy counts and stellar colour-colour plots (see below), we estimate that our absolute photometric solution in each filter has a maximum systematic error of $\sim 0.05$ mag. Catalogues were extracted using SExtractor in dual-image mode, with detections carried out using a chi-squared image (Szalay et al. 2003) constructed from the $g^{\prime} r^{\prime} i^{\prime}$ images. Kron-like total magnitudes were used

\footnotetext{
${ }^{2}$ http://terapix.iap.fr/article.php?idarticle $=383$

${ }^{3}$ http://terapix.iap.fr/article.php?idarticle $=382$
} 

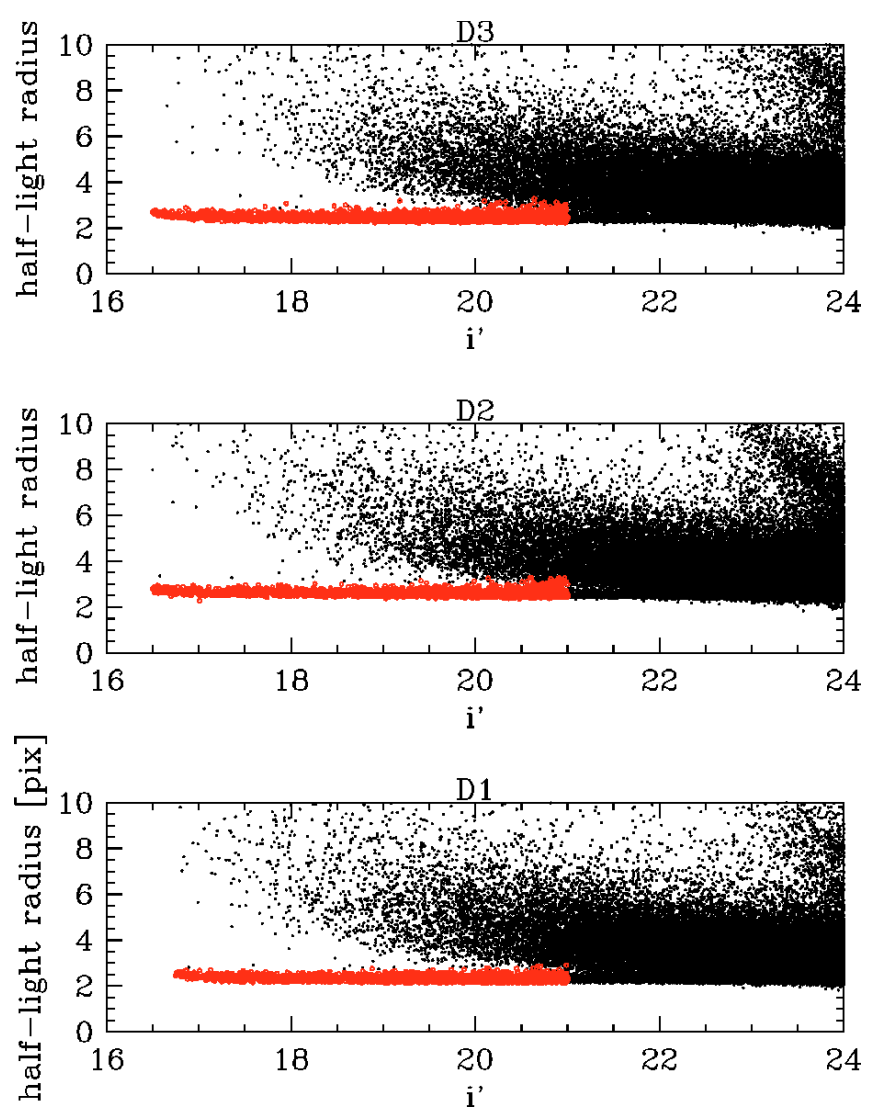

Fig. 1. $i^{\prime}$ magnitude vs half-light radius (in pixels) for the D1, D2 and D3 field. The filled circles show the selected stellar branch. Note that objects with $i^{\prime}<17$ are saturated.

throughout. Through this paper, our magnitudes are presented in the MEGACAM instrumental AB system.

\section{Star-Galaxy separation}

We separated point-like sources from extended ones using SExtractor's (Bertin \& Arnouts 1996) "flux-radius" parameter measured on the $i^{\prime}$-band image. This parameter measures the radius which encloses $50 \%$ of the object's flux: for point-like sources this is independent of magnitude, and depends only on the image FWHM. The stars were selected by automatically locating the stellar branch in the flux-radius-magnitude diagram in a series of 10 arcminute cells distributed over each MEGACAM $i^{\prime}$-stack, which accounts for variation of $F W H M$ over MEGACAM field of view. Figure 1 shows the compactness parameter against the magnitude for the three CFHTLS fields D1, D2 and D3. At magnitudes fainter than $i^{\prime}=21.0$ the separation between stars and galaxies starts to be problematic. From Fig. 1 it is clear that for the D1 field the contamination of galaxies is small for $i^{\prime}<21.0$ while for the D2 and the D3 field the star/galaxy separation starts to fail at already $i^{\prime} \sim 20.5$. The choice of the cut-off at $i^{\prime}=21.0$ for the T0001 release is certainly a conservative criterion which can not be applied for all three fields. However, the star/galaxy separation depends on the colour of the objects. If one restricts to red objects with $r^{\prime}-i^{\prime}>1.4$, stars can be better seperated from galaxies and thus stars can be extracted until $i^{\prime}<22.5$ (for the D1 field). We discuss and quantify below galaxy contamination as a function of magnitude and colour.

In order to assess the number of galaxies contaminating our stellar sample, we used spectroscopic data from the VIMOSVLT Deep Survey (VVDS). The VVDS (Le Fèvre et al. 2005) intends to measure redshifts over $0<z<5$ across $16 \mathrm{deg}^{2}$ in four separate fields. The survey is conducted in two steps: an imaging survey and a spectroscopic survey. Deep imaging in the UBVRI bands and partly $K^{\prime}$ band is obtained with the CFHT, ESO-NTT and ESO-2.2 m. The VLT VIMOS instruments allow the measurement of redshifts of objects selected from the imaging survey. The so-called "Deep" survey has a limiting magnitude $I_{A B}=24$. Its location, $\alpha=2 \mathrm{~h} 26 \mathrm{~m}$ and $\delta=-4^{\circ} 30^{\prime}$ overlaps with that of the D1 field. For a detailed description of the VVDs data we refer to Le Fèvre et al. (2005). We extended the limiting magnitude for the star/galaxy separation of the D1 field to $i^{\prime}=22.0$ mag (thus 1 mag deeper than the official T0001 release), using the half light radius, as described above. The D1 stacks have better seeing than the other fields, allowing star-galaxy separation to fainter magnitudes. We use this deeper catalogue in order to discuss the galaxy contamination (see below). However, for the rest of the paper we use the official T0001 release, in which stars are separated from galaxies only until $i^{\prime}=21.0$.

We cross-identified the D1 data with the VVDS data using a search radius of $2^{\prime \prime}$. Due to the masking and only partial overlap between the D1 field and the VVDS F02-field, only an area of $0.4 \mathrm{sq}$ degree is in common. Out of 9088 sources of VVDS data, 7110 sources have been cross-identified with the D1 field. 295 sources were identified as stellar and are shown in Fig. 2.

Figure 2 shows the colour-colour diagrams of the D1 field cross-identified with the VVDS data. All objects shown are classified as stars from the morphological criterion. The dots show the spectroscopically identified stars and asterisks those objects spectroscopically identified as galaxies. The majority of the galaxies classified morphologically as stars populate the blue part of the colour-colour diagram with $r^{\prime}-i^{\prime}<0.4$. They populate the stellar locus as well as regions outside of the stellar sequence. Table 2 gives the percentage of galaxies contaminating the stellar sample as a function of $i^{\prime}$ magnitude and $r^{\prime}-i^{\prime}$ colour. The percentage of galaxy contamination depends very much on the $r^{\prime}-i^{\prime}$ colour, which means that going to redder colour diminishes significantly the contamination by galaxies. For $r^{\prime}-i^{\prime}<0.5$ and $i^{\prime}<22$ one obtains for the D1 field a rate of $23.5 \%$ of contaminating galaxies while for $r^{\prime}-i^{\prime}>1.4$ the percentage of galaxy contamination is negligible. Note that the galaxy contamination is larger for the D2 and D3 fields.

In addition, we visually inspected (for the D1 field) sources which are located outside the stellar locus. We have marked them in Fig. 2 as open squares. Most of these objects are galaxies blended with stars where obviously the aperture photometry is unreliable. Note that only the $g^{\prime}-r^{\prime}$ vs. $r^{\prime}-i^{\prime}$ diagram reveals these blends easily. 

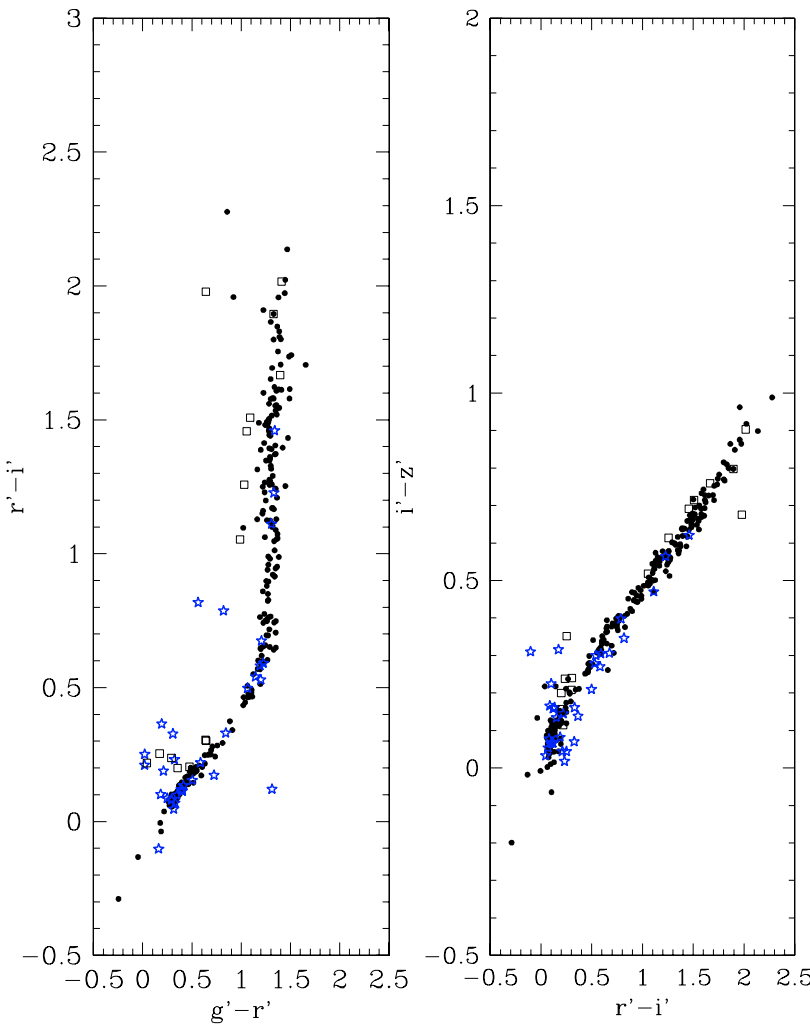

Fig. 2. Colour-colour diagrams of the D1 field cross-identified with the VVDS data. Objects shown are all morphologically classified as stars. Dots are spectroscopically identified stars. Asterisks are galaxies and open squares indicate sources where a blend of a galaxy with a star has been found by visual inspection of the images. The limiting magnitude used for the morphological star/galaxy separation is $i^{\prime}=$ $22.0 \mathrm{mag}$.

\section{Stellar atmospheres and the theoretical stellar locus}

Using the published response of the CFHT, Megaprime and MEGACAM we have computed synthetic colours of the stars as a function of temperature, gravity and metallicity. To do this we have used two sets of stellar atmosphere models: Basel 3.1 and NextGen. The Basel3.1 library is a semi-empirical library based on preceding generation of models, Basel 2.2 (Lejeune et al. 1997), extended to non-solar metallicities by Westera et al. (2002). The Kurucz theoretical spectra (1979) have been modified to fit broad band photometry using the algorithm by Cuisinier et al. (see Buser \& Kurucz 1992). The corrected spectra are used for integrating the flux in the desired bands. NextGen is the 1997 version of atmosphere models from Allard et al. (1997). These models use a direct opacity sampling including over 500 million lines of atomic and molecular species. They give a more realistic description of the M dwarf population.

Figure 3 shows the CFHTLS colour-colour diagrams of the D1 field superimposed with the synthetic colours of dwarf stars using the Basel3.1 stellar library for solar metallicity, $[\mathrm{Fe} / \mathrm{H}]=$ -1.0 and $[\mathrm{Fe} / \mathrm{H}]=-2.0$ (right panel) and the NextGen library for $[\mathrm{Fe} / \mathrm{H}]=0.0$ and $[\mathrm{Fe} / \mathrm{H}]=-1.0$ (left panel). For a better definition of the stellar locus, we use here only stellar objects
Table 2. Galaxy contamination of the D1 field cross-identified with the VVDS field as a function of $i^{\prime}$ magnitude and $r^{\prime}-i^{\prime}$ colour. The common area is $\sim 0.4$ sq degree.

\begin{tabular}{rccc}
\hline \hline$r^{\prime}-i^{\prime}$ & $i^{\prime}<20.0$ & $i^{\prime}<21.0$ & $i^{\prime}<22.0$ \\
\hline total & $3.2 \% \pm 1.8$ & $6.7 \% \pm 1.9$ & $13.1 \% \pm 2.3$ \\
$<0.5$ & $3.2 \% \pm 3.2$ & $13.0 \% \pm 4.3$ & $23.5 \% \pm 4.9$ \\
$>0.5$ & $3.1 \% \pm 2.2$ & $2.7 \% \pm 1.5$ & $6.5 \% \pm 2.1$ \\
$>1.4$ & $0 \%$ & $0 \%$ & $1.8 \% \pm 1.8$ \\
\hline
\end{tabular}

which have a photometric error estimate smaller than $0.01 \mathrm{mag}$ in each filter. We noted that the $i^{\prime}-z^{\prime}$ colour has a slight offset of 0.05 mag compared to the model which comes from uncertainties on the photometric calibration. For the D2 and D3 field we noted a shift of $0.07 \mathrm{mag}$ and $0.02 \mathrm{mag}$ in $r^{\prime}-i^{\prime}$ respectively. These offsets have been applied.

Figure 3 illustrates the sensitivity of the colours in the CFHTLS system to metallicity and the differences in the stellar libraries.

For temperatures below $3500 \mathrm{~K}$, which correspond to $\mathrm{K} / \mathrm{M}$ stars, the Basel3.1 library does not give realistic colours for cool dwarfs, but gives a better fit to the data than NexGen models do for hotter stars.

In the temperature range 7000 to $4000 \mathrm{~K}$, the most sensitive colour is $g^{\prime}-r^{\prime}$, whereas for cooler stars this colour index saturates and $r^{\prime}-i^{\prime}$ becomes better. The $i^{\prime}-z^{\prime}$ colour seems to be redundant with $r^{\prime}-i^{\prime}$, but going to very cool stars we expect it to be a very good indicator for selecting brown dwarfs (see Sect. 7.2).

Both atmosphere models show a strong metallicity effect for cool dwarfs in $g^{\prime}-r^{\prime}$ and $i^{\prime}-z^{\prime}$. It appears that at $g^{\prime}-r^{\prime}>1$ this index is no longer sensitive to temperature but to metallicity. If the photometric calibration of the survey is accurate and the model atmospheres reliable, we will be able to constrain the metallicity distribution of these cool stars, at least statistically. This will permit us to determine the metallicity range, and probably the thin disc to thick disc density ratio, as we expect a difference of metallicity of about 0.5 dex between these two populations, corresponding to about 0.15 magnitude in $g^{\prime}-r^{\prime}$ at a temperature of $4000 \mathrm{~K}$.

\section{The Besançon Galaxy model}

The Besançon Galaxy model is a simulation tool aimed at testing galaxy evolution scenarii by comparing stellar distributions predicted by these scenarii with observations, such as photometric star counts and kinematics. A complete description of the model ingredients can be found in Robin et al. (2003). We summarise here the model's principal features.

The model assumes that stars are created from gas following a star formation history and an initial mass function; stellar evolution follows evolutionary tracks. To reproduce the overall galaxy formation and evolution we distinguish four populations of different ages and star formation history, which we now describe.

The oldest population is the spheroid. For this population we assume a single-burst star formation history ocurring early in the lifetime of the Galaxy, around $14 \mathrm{Gyr}$ ago, from gas still 

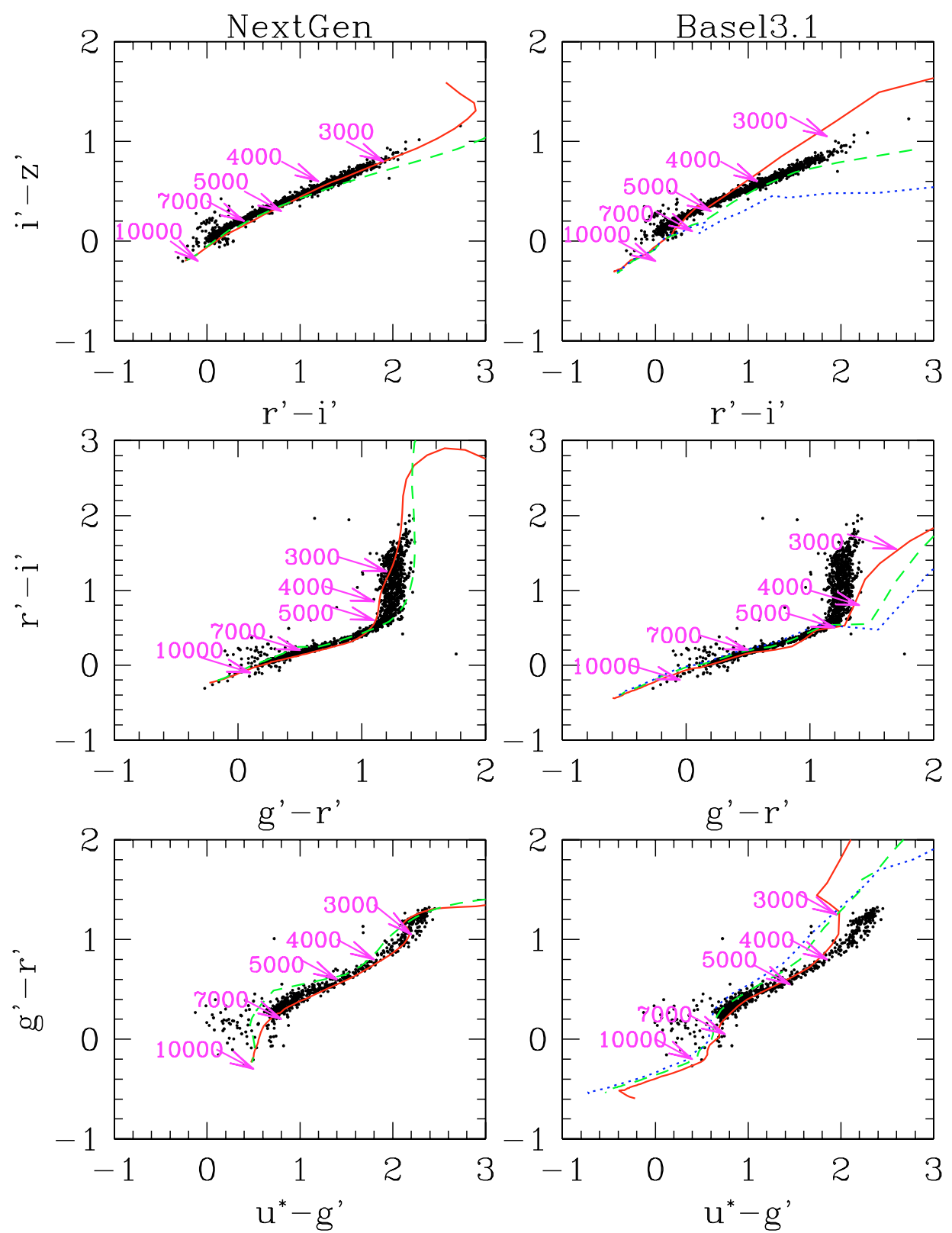

Fig. 3. Colour-colour diagrams of the D1 field compared to the synthetic colour-colour diagram of NextGen (left panel) and Basel3.1 (right panel) for different metallicities. The solid line denotes $[\mathrm{Fe} / \mathrm{H}]=0.0$, the dashed line $[\mathrm{Fe} / \mathrm{H}]=-1.0$ and the dotted line $[\mathrm{Fe} / \mathrm{H}]=-2.0$. For a better determination of the stellar locus, we show here only stellar objects with a photometric error smaller than 0.01 mag in each filter. The approximate temperatures are also indicated.

in a spheroidal distribution. The initial mass function (IMF) and the density distribution of this population is characterised by a power law index, an axis ratio and a local normalisation, which are constrained by remote star counts at high and medium Galactic latitudes (Robin et al. 2000). The kinematics are also deduced from in situ velocity determinations. The mean metallicity of the spheroid is assumed to be $-1.7 \mathrm{dex}$ with an intrinsic dispersion of $0.5 \mathrm{dex}$.

Secondly, a population, called the thick disc, is formed of stars born about 11-12 Gyr ago in a short period of time as implied by recent metallicity determinations for this population. We also assume a single burst for simplicity. For the thick disk, star formation occurred from the gas already settled in the disk.
The kinematics, deduced from observational constraints (Ojha et al. 1996; Ojha 1999), implies that it has undergone a merging event shortly after the disc formation (Robin et al. 1996), increasing the disk thickness and giving a higher velocity dispersion and scale height. The IMF, density distribution and local normalisation were constrained from star counts (Reylé \& Robin 2001). The mean metallicity of the thick disc is assumed to be -0.7 dex with an intrinsic dispersion of $0.3 \mathrm{dex}$.

Thirdly, a bulge population is present in the center of the Galaxy and extends to about $2 \mathrm{kpc}$. Its age is of the order of 10 Gyr. This value is however poorly constrained. This population has a triaxial distribution, as a bar as determined by Picaud et al. (2004) from near-infrared star counts. Velocity 
dispersions are large, similar to the spheroid. The mean metallicity is assumed solar with a dispersion of 0.5 dex.

A standard evolution model is used to compute the disc population, based on a typical set of parameters: an IMF, a star formation rate (SFR), a set of evolutionary tracks (see Haywood et al. 1997a, and references therein). The disc population is assumed to evolve during 10 Gyr. A set of IMF slopes and SFR's are tentatively assumed and tested against star counts. The tuning of disc parameters against relevant observational data has been described in Haywood et al. (1997a,b).

A revised IMF has been used as a starting point in the present analysis, adjusted to agree with the most recent Hipparcos results: the age-velocity dispersion relation is from Gómez et al. (1997), the local luminosity function is from Jahreiß \& Wielen (1997) and an IMF is adjusted to it, giving an IMF slope $\alpha=1.5$ in the low mass range [0.5-0.08 $M_{\odot}$ ], in good agreement with Kroupa (2001). The scale height has been computed self-consistently using the potential via the Boltzmann equation. The local dynamical mass was taken from Crézé et al. (1998).

The evolutionary model fixes the distribution of stars within the parameter space of effective temperature, gravity, absolute magnitude, mass and age. These parameters are converted into colours in various systems through stellar atmosphere models.

In the case of the MEGACAM photometric system, we have used the optics, CCD and filter definition of the passbands, and applied them to the spectral libraries. As seen in Fig. 1, the Basel3.1 library is more suitable for hot stars, giving better predicted colours, especially $u^{*}-g^{\prime}$, while the NextGen library is more realistic for cool stars. Hence we have adopted a combination of both: Basel3.1 at $T_{\text {eff }}>4000 \mathrm{~K}$ and NextGen for cooler stars. As the cooler stars are mostly located in the disc, we used only $[\mathrm{Fe} / \mathrm{H}]=0.0$ and $[\mathrm{Fe} / \mathrm{H}]=-1.0$ for $T_{\text {eff }}<4000 \mathrm{~K}$. The model simulations also include a model of extinction and account for observational errors. The Besançon model predictions in the MEGACAM photometric system can be found at http: //www . obs-besancon. fr/modele

\section{Stellar populations}

Simulations from the Besançon model in the MEGACAM photometric system have been performed and compared with the CFHTLS in three fields: D1, D2 and D3.

We used the same magnitude limit in $i^{\prime}$ of 21.0 imposed by the star/galaxy separation and took the photometric errors in each band as a function of magnitude into account. In the discussion below we indicate also the different components of the Galactic model such as thin disc, thick disc and the spheroid. Figure 4 shows the expected colour-colour diagram predicted by the Besançon model for the D1 field. Indicated are the three different components, thin disc, thick disc and spheroid. While the blue part of the colour-colour diagram is populated by mainly spheroid stars, the thick disc stars are concentrated around $0.6<r^{\prime}-i^{\prime}<0.9$ and the thin disc stars cover the red part of the diagram.

Figure 5 shows the colour-colour diagrams of the CFHTLS for the D1, D2 and D3 fields respectively as well as the model

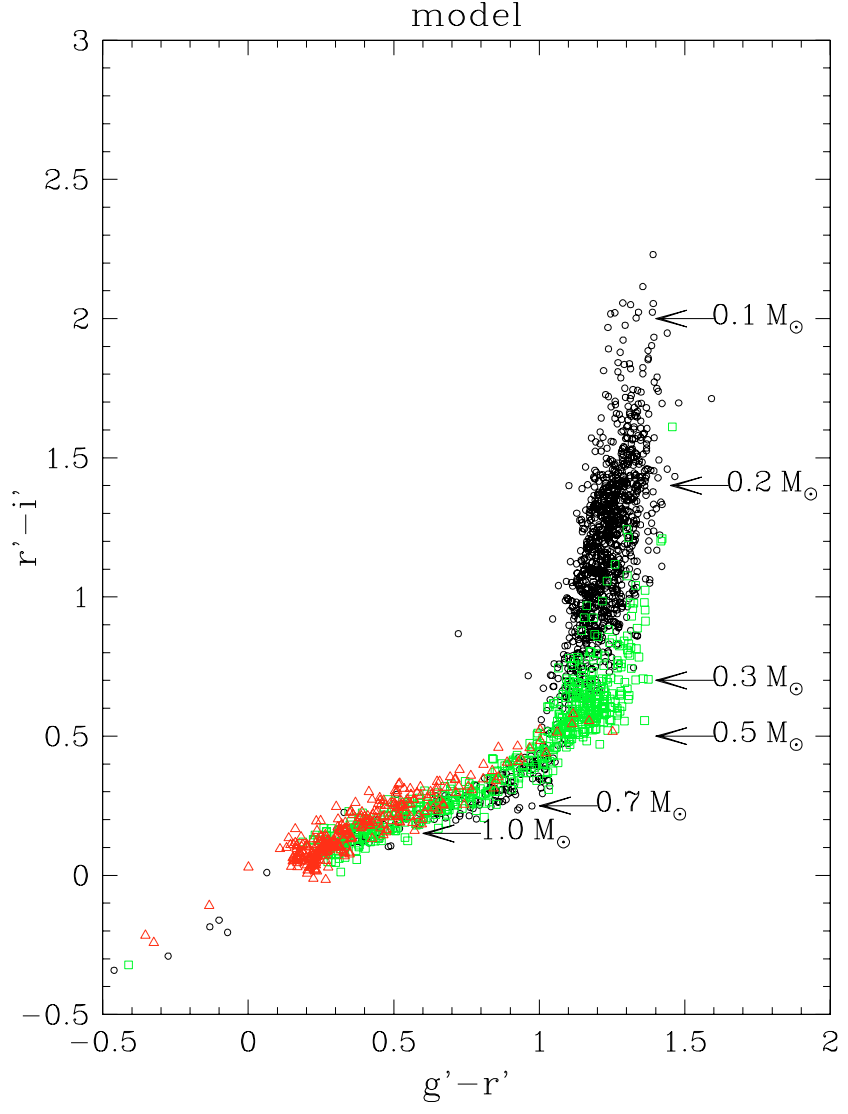

Fig. 4. Colour-colour diagram predicted by the Besançon model for the D1 field for thin disc (dots), thick disc (open squares) and spheroid (asterisks) stars.

predictions. Note the excellent overall agreement between observed and predicted colours for the three Galactic components. Figure 5 also shows that the stellar populations of the three deep fields are similar, although one notices several differences:

- there is a steady increase in the width of the stellar locus from the D1 field - which has a well defined narrow locus - towards the D3 field - which is wider - and the D2 field which is wider still, especially at $r^{\prime}-i^{\prime}>1.2$, and more pronouced in $i^{\prime}-z^{\prime}$.

- The blue part of the colour-colour diagrams $\left(g^{\prime}-r^{\prime}<0.5\right.$, $\left.r^{\prime}-i^{\prime}<0.4, i^{\prime}-z^{\prime}<0.3\right)$ is populated by an overdensity of objects which has a larger spread than the predicted stellar locus. These objects are compact faint galaxies or quasars erroneously classified as stars by morphological criteria (see Sect. 4). This contamination is larger in D2 and D3 than in D1.

- In the $g^{\prime}-r^{\prime}$ vs $r^{\prime}-i^{\prime}$ diagram, there are sources with $g^{\prime}-r^{\prime}>1.3$ and $r^{\prime}-i^{\prime}<1.5$ abundant in the D2 and D3 fields, but absent in the D1 field. Pello (priv. communication) provided us with theoretical colours of galaxies in the MEGACAM photometric system for different redshifts and for different morphological types which show that these objects are expected to be elliptical galaxies with $z<0.5$ while a few high redshift QSOs and spiral galaxies could also be present. 

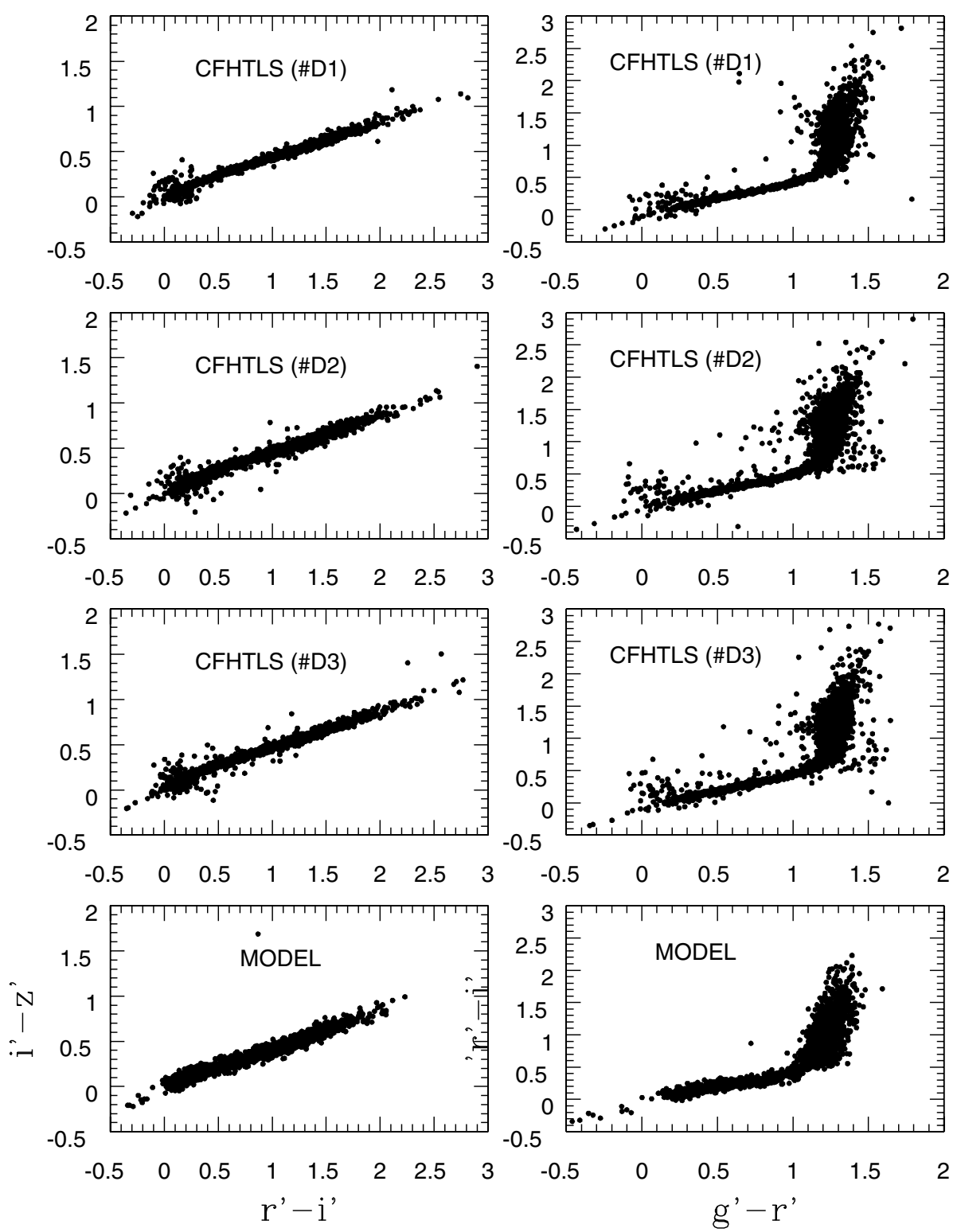

Fig. 5. Colour-colour diagrams of the three Deep field D1, D2 and D3 compared with synthetic colours predicted by the Besançon model (lower panel). Note the excellent agreement between the data and the model.

- In the D1, D2 and D3 fields there are objects with $r^{\prime}-i^{\prime}>2$ and $i^{\prime}-z^{\prime}>1.2$ which are off the stellar sequence which may be brown dwarf candidates (see Sect. 7.2), or high redshift quasars.

- The dispersion in the synthetic colour-colour diagram is larger than observed, especially in the blue part. This is due to uncertainties of the model atmospheres in this metallicity range, as seen in Fig. 3.

\subsection{White dwarfs}

White dwarfs (WD) are the last stage of stellar evolution and their space density depends on the Galactic star formation history and initial mass function. While the luminosity function (thereafter LF) of white dwarfs in the thin disc is known from systematic searches in the solar neighbourhood (e.g. Liebert et al. 1988; Ruiz \& Bergeron 2001; Holberg et al. 2002), only very few thick disc white dwarfs have been identified while the presence of white dwarfs in the Galactic halo is still uncertain. Knowledge of the luminosity function of thick disc and halo white dwarfs is expected to constrain the age of these populations, the physics of the coolest white dwarfs, as well as the initial mass function at early epochs in the Galaxy (Chabrier 2003).

Crézé et al. (2004) used two-epoch observations of the $1 \mathrm{deg}^{2}$ VVDS-F02 deep field to search for white dwarfs in the VVDS survey by proper motions; they reported a null detection.

Bergeron (priv. communication) provided us with theoretical colours of white dwarfs in the MEGACAM photometric system. Figure 6 shows their location in various colour-colour diagrams compared with data in the D1 field. The locus of the white dwarfs is distinct from the locus of the subdwarfs in the $u^{*}-g^{\prime}$ vs. $g^{\prime}-r^{\prime}$ plane only. Furthermore, we note the sensitivity 

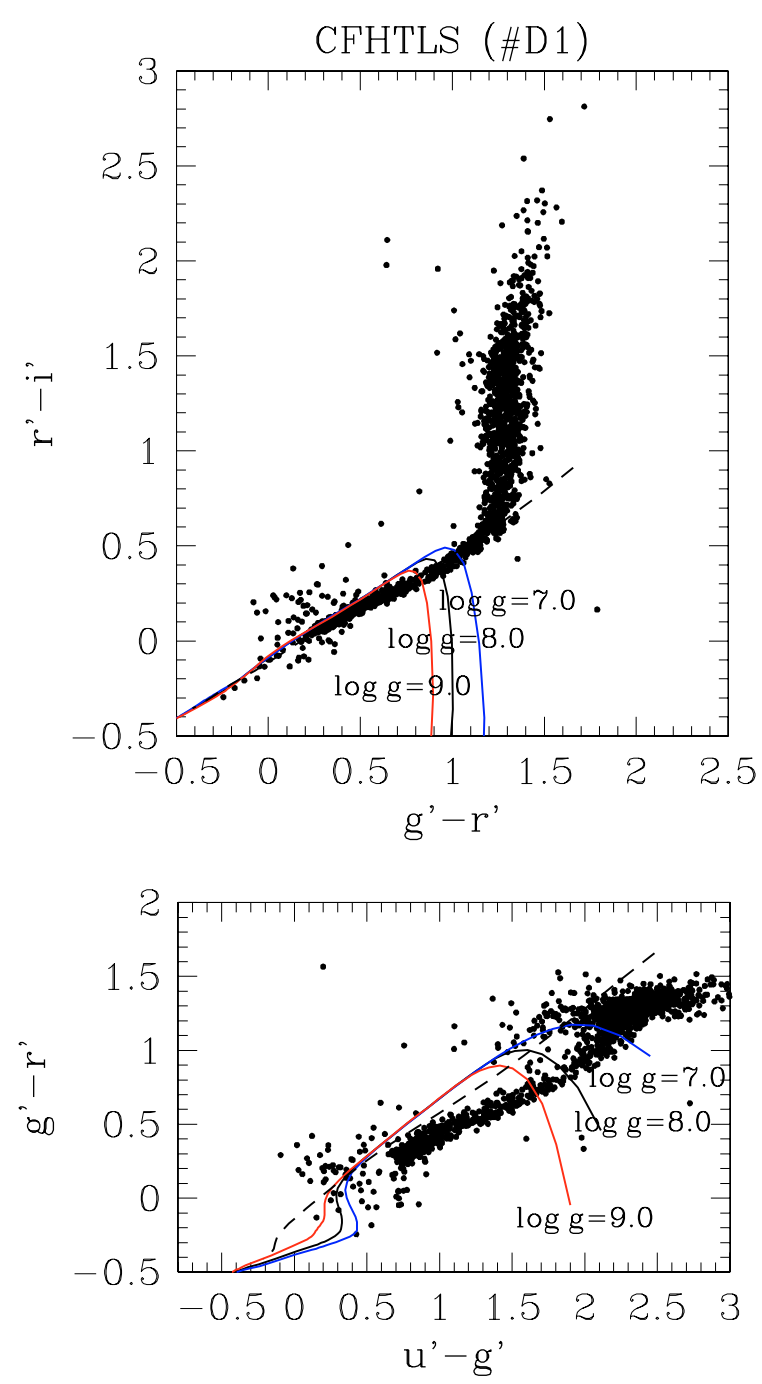

Fig. 6. White dwarf sequences in colour-colour diagrams of the D1 field. Solid line shows the DA sequence, dashed line the DB sequence from theoretical model from Bergeron et al. (2001) for surface gravities of $\log g=7.0,8.0$ and 9.0.

of the colours to $\log g$. However this part of colour-colour space is highly contaminated by quasars. In the other colour combinations, white dwarfs are indistinguishable from subdwarfs, except the very cool ones (temperature less than about $3200 \mathrm{~K}$ ) where DA white dwarfs with hydrogen atmospheres start to deviate from blackbody.

In the present data, there are a few objects with colours consistent with those of white dwarfs. However these objects could also be horizontal branch spheroid stars or quasars. White dwarf candidates will only be reliably identified when proper motions become available. Proper motions will easily distinguish these objects from horizontal branch stars, as they are brighter, hence much more distant at a given apparent magnitude (extragalactic objects such as quasars, of course, will have no measurable proper motions). The CFHTLS will allow us to eventually cover about 150 square degrees to the same magnitude limit with proper motions, allowing a definitive answer if baryonic matter is present in galactic halos in the form of white dwarfs.

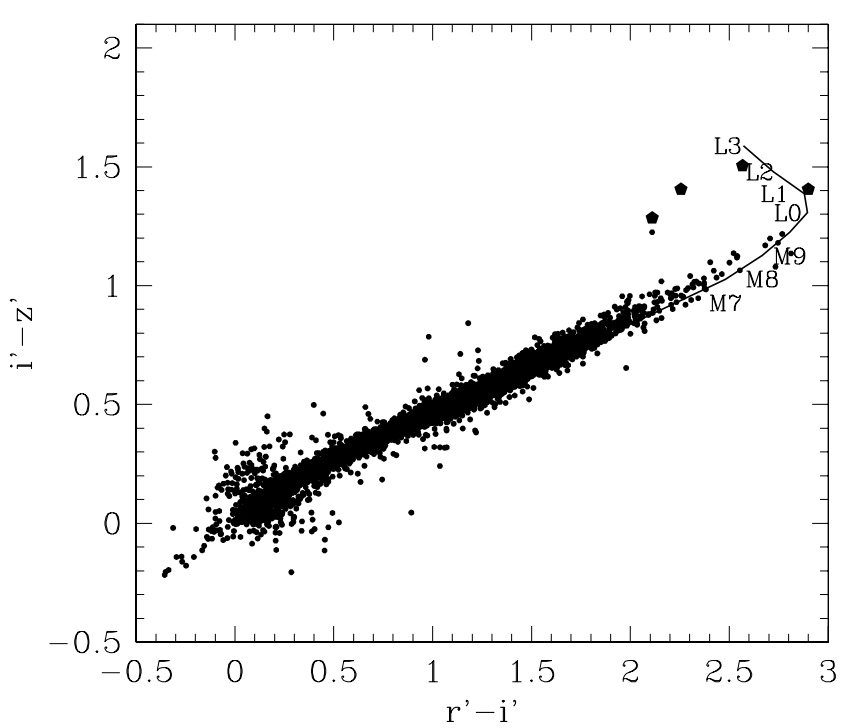

Fig. 7. $r^{\prime}-i^{\prime}$ vs. $i^{\prime}-z^{\prime}$ diagrams of the D1, D2 and D3 fields. Superimposed are the average colors of $\mathrm{M}$ and $\mathrm{L}$ dwarfs taken from NextGen models. Spectral types are indicated, following the temperature-spectral type relation from Golimowski et al. (2004). Candidate $\mathrm{L}$ dwarfs are shown as asterisks (see also Table 3 ).

Table 3. Positions and MEGACAM photometry of late type dwarfs or high redshift quasars.

\begin{tabular}{cccccc}
\hline \hline Field & RA (J2000) & Dec (J2000) & $i^{\prime}(\mathrm{mag})$ & $r^{\prime}-i^{\prime}$ & $i^{\prime}-z^{\prime}$ \\
\hline D1 & 2h24m58.2s & $-4^{\circ} 17^{\prime} 00.3^{\prime \prime}$ & 20.43 & 2.11 & 1.28 \\
D2 & $9 \mathrm{~h} 59 \mathrm{~m} 4.1 \mathrm{~s}$ & $+2^{\circ} 34^{\prime} 27.9^{\prime \prime}$ & 19.66 & 2.90 & 1.40 \\
D3 & $14 \mathrm{~h} 19 \mathrm{~m} 53.4 \mathrm{~s}$ & $+52^{\circ} 17^{\prime} 52.9^{\prime \prime}$ & 20.39 & 2.56 & 1.51 \\
D3 & $14 \mathrm{~h} 22 \mathrm{~m} 01.6 \mathrm{~s}$ & $+52^{\circ} 48^{\prime} 46.6^{\prime \prime}$ & 20.57 & 2.26 & 1.40 \\
\hline
\end{tabular}

The number of expected white dwarfs per square degree, as predicted by the Besançon Galaxy model is about 25 for the thin disc, around two for the thick disc and about 0.1 for the halo, to magnitude $i^{\prime}=22.5$. The number of ancient halo WDs is computed assuming that the dark halo is partly made of ancient white dwarfs, at the level of $2 \%$ of its mass density. Even at these bright magnitudes their identification from photometry only will be difficult due to the large number of galaxies and quasars; proper motions are necessary. Assuming that the survey would reach an astrometric accuracy of about 0.1 pixel at $i^{\prime}=22.5$, with a time baseline of three years, objects having a motion of $25 \mathrm{mas} / \mathrm{yr}$ would be detectable at the 3- $\sigma$ level. About 2/3 of the disc WD pass this proper motion selection criterion, $80 \%$ of the thick disc WDs, and $100 \%$ of the halo WDs.

\section{2. $M, L$, and $T$ dwarfs}

Large sky surveys are very efficient in identifying extremely late-type stellar and sub-stellar objects because they are detectable only at short distances from the sun, and hence in a small volume. Even if they are numerous, the number of brown dwarfs detectable to date is still small. Near-infrared surveys such as DENIS and 2MASS resulted in the detection of a significant number of close low-mass stars and brown dwarfs (see 
e.g. Kendall et al. 2004; Reid et al. 2002; Burgasser et al. 2004). The SDSS survey was used to identify the first field T dwarfs (Strauss et al. 1999; Tsvetanov et al. 2000) and the numbers of known L dwarfs has been greatly increased (Fan et al. 2000; Schneider et al. 2002). Gelino et al. (2004) introduced an homogeneous database of M, L, and T dwarfs, that contains more than one thousand of these objects. Hawley et al. (2002) compiled a large sample of M, L and T dwarfs from SDSS spectra together with SDSS photometry and additional near-IR photometry (2MASS). They find that the $i-z$ and $i-J$ colour are the most useful for estimating spectral types based solely on photometric information for $\mathrm{M}$ and $\mathrm{L}$ dwarfs.

Figure 7 shows the $i^{\prime}-z^{\prime}$ vs. $r^{\prime}-i^{\prime}$ diagram of the three CFHTLS fields together superimposed with the NextGen model at solar metallicity. The spectral types are from the temperature-spectral type relation from Golimowski et al. (2004). There are two objects with $r^{\prime}-i^{\prime}>2$ and $i^{\prime}-z^{\prime}>1.3$ in the D3 field and one candidate each in the D1 and D2 fields, which are good candidates for being either early L dwarfs or high redshift quasars (see Fig. 7). They are given in Table 3. High redshift quasars with $i^{\prime}-z^{\prime}>1.5$ will be distinguishable from brown dwarfs either by near-infrared photometry or by proper motion measurements.

\subsection{Binary systems}

The formation and evolution of low-mass stars in a binary system is a common phenomenon which leads to the interesting class of cataclysmic variables. In deep surveys one expects to detect a few cases of WD-M dwarf pairs. Raymond et al. (2003) identified $\sim 100$ white dwarf-M dwarf pairs in the SDSS survey with $g<20$. Using additional spectroscopy, they achieve an efficiency of $\sim 60 \%$ in finding white dwarf-M dwarf pairs because of the contamination by galaxies in the interesting colour regions.

We simulated a sample of unresolved $\mathrm{M}$ dwarfs + white dwarfs systems by merging their fluxes. Typical colours of these simulated systems are given in Fig. 8 as star symbols. The location of these systems is clearly outside the single star locus in the $g^{\prime}-r^{\prime}$ vs. $r^{\prime}-i^{\prime}$ diagram. However they lie in a region where we expect contamination by compact galaxies and quasars. Their identification will be easy using proper motions, all these objects being intrinsically faint, and are hence detected only in the solar neighbourhood.

\section{Stellar densities and the IMF at low masses}

As mentioned above, the stellar samples are contaminated by non-stellar sources. As a large fraction of those fall inside the stellar locus and stellar binaries and white dwarfs are also expected outside the main sequence (see Fig. 2), only proper motions can be used to clean our sample and remove galaxies and quasars. In the following all objects classified as stellar are kept.

In Fig. 9 histograms in $g^{\prime}-r^{\prime}, r^{\prime}-i^{\prime}$ and $i^{\prime}-z^{\prime}$ for the D1 field are shown, with model predictions for each population, thin disc, thick disc, and spheroid. Model predictions are acceptable
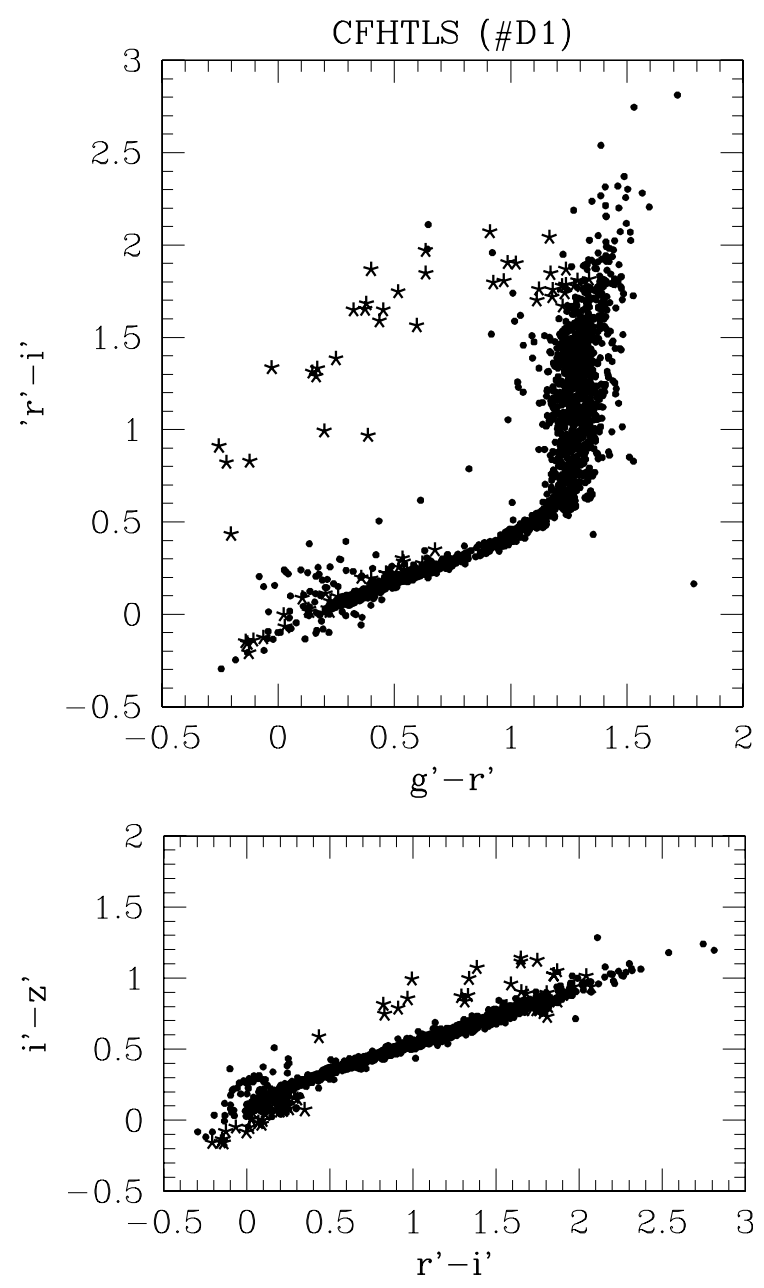

Fig. 8. Binary systems M dwarf + White dwarf in the D3 field. Dots: whole D1 data set. Stars: simulated systems. Colours are estimated by adding up the flux in a realistic sample of unresolved systems.

for all three populations, which are better separated in the $r^{\prime}-i^{\prime}$ colour.

\subsection{Stellar densities}

Figure 10 shows the $g^{\prime}-r^{\prime}$ vs. $r^{\prime}-i^{\prime}$ diagram for each CFHTLS field, compared with model predictions, where the greyscale indicates the number of stars. The colour-colour diagrams in the three fields are similar, and model predictions are in good general agreement with the data. We notice, however, a few significant differences:

- In all three fields the predicted source density is a slightly low compared with the observations. The difference between the data and the predicted colour counts is of order $\sim 30 \%$ for $r^{\prime}-i^{\prime}<0.3$. We estimate that part of the excess in the data is overestimated, due to the contamination by galaxies, which is in the blue $\sim 13 \%$ for the D1 field but larger for the D2 and D3 field (see also Sect. 4). On the other hand, the predicted counts of spheroid stars depend on three density parameters: the axis ratio, the power law exponent and the local normalization. These parameters have been already constrained by other data sets 

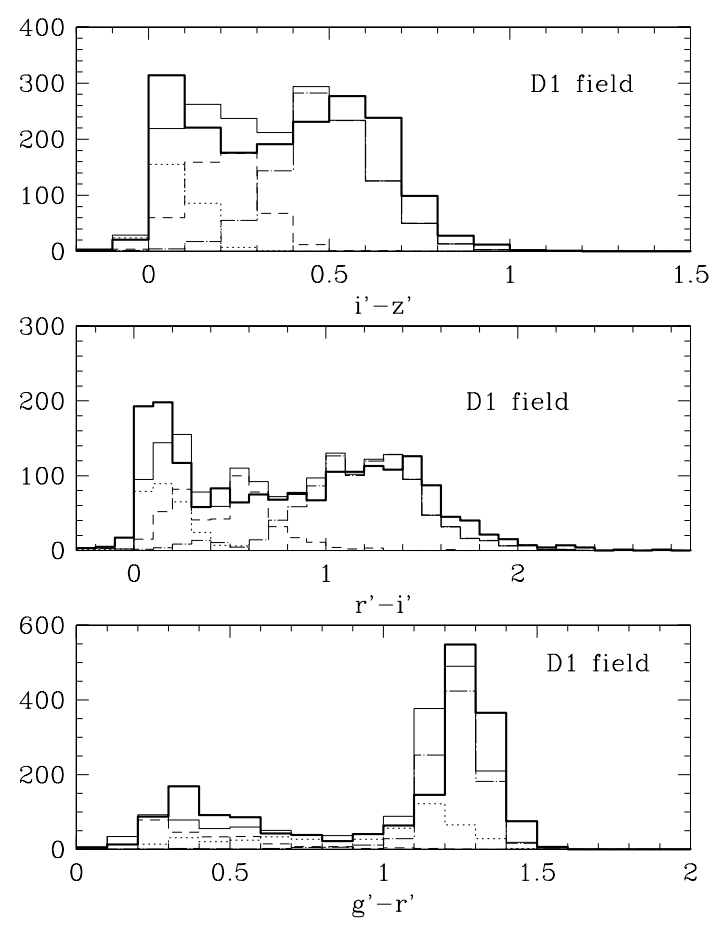

Fig. 9. Histograms of the colour distributions in the D1 field. The thick line denotes the observations, the thin line the Besançon model, the dotted line the contribution of the spheroid, the short-dashed line the thick disc and the dot-long dashed line the thin disc contribution.

(Robin et al. 2000) but could be better adjusted using new large and deep surveys such as the CFHTLS or SDSS. In order to perform better adjustments of these parameters, we need more fields with wider areas of the sky than those presented here. The effects on the star counts of the derived IMF of this population as well as their density distribution can only be disentangled from large surveys in different areas of the sky. This will be considered in a future paper using the T0002 and T0003 releases.

- In the range $0.3<r^{\prime}-i^{\prime}<0.7$ the thick disc population dominates while for $r^{\prime}-i^{\prime}>0.8$ the thin disc dominates the counts. The whole colour range is well modeled in the three fields; the thick disc model fitted in Reylé \& Robin (2001) fits well here too, in addition to the thin disc for $0.8<r^{\prime}-i^{\prime}<1.4$.

- In the very red part of the diagram, at $r^{\prime}-i^{\prime}>1.6$, the number of observed stars is significantly larger than predicted by the model. We do not expect large numbers of very high redshift galaxies and quasars at these colours. These point source objects are most probably disc M dwarfs. This implies that the assumed luminosity function of these stars has been underestimated in the model. We investigate this point further below.

\subsection{The IMF at low masses}

The behaviour of the stellar luminosity function (LF) and mass function $(\mathrm{MF})$ for low-mass stars $\left(<1 M_{\odot}\right)$ is still under debate. Jahreiß et al. (1997) derived a local stellar LF (within 20 pc) from the Catalogue of Nearby Stars revised with Hipparcos
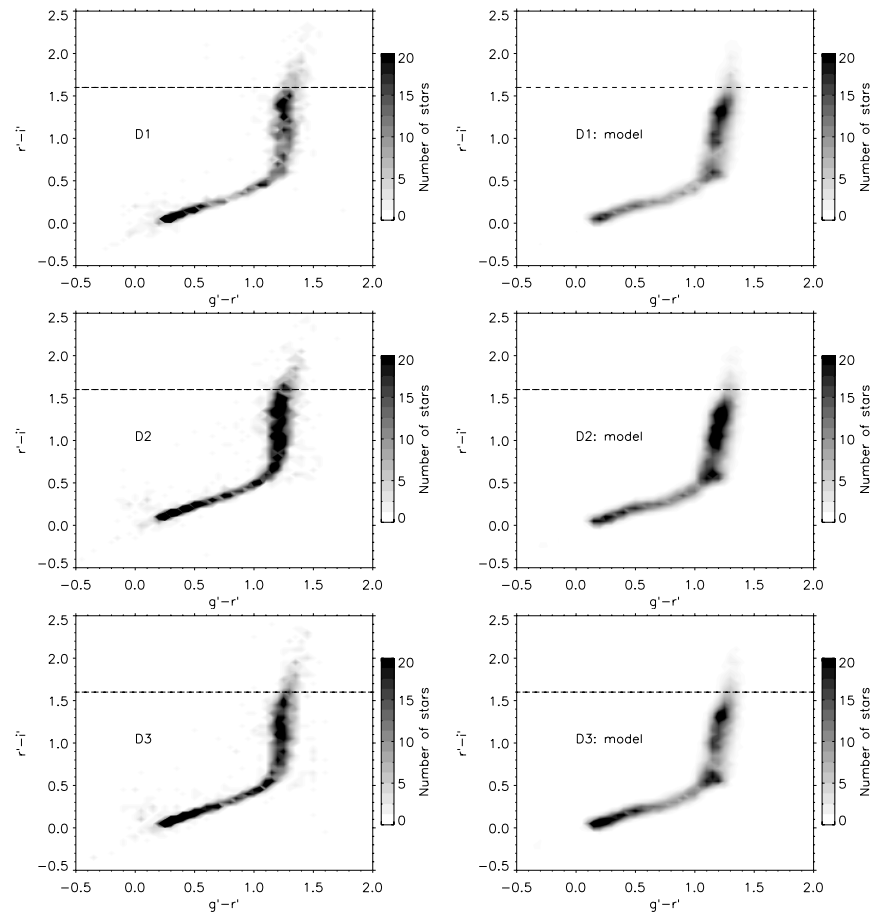

Fig. 10. $g^{\prime}-r^{\prime}$ vs. $r^{\prime}-i^{\prime}$ diagram of the D1, D2 and D3 field compared to the synthetic colours predicted by the Besançon model, all populations included. The greyscale indicates the number of stars per bin width of 0.05 mag in each colour.

data. Reid et al. (2002) derived the nearby star LF using the Palomar/Michigan State University sample (PMSU) combined with the Hipparcos $25 \mathrm{pc}$ sample. Both LFs are shown in Fig. 11. Error bars are large due to the small survey volumes. For masses smaller than $0.5 M_{\odot}$, the determination of the MF is hampered by the incompleteness of the different samples (Henry et al. 1997; Chabrier \& Baraffe 2000) and by the unknown proportion of M dwarfs in binaries. Chabrier (2003) estimated that the mass function below $1 M_{\odot}$ is consistent with a fraction of $\sim 50 \%$ of $\mathrm{M}$ dwarf binaries where $30 \%$ should have an M dwarf companion and $20 \%$ a brown dwarf secondary.

Also in Fig. 11 are superimposed the luminosity functions used for simulations with our standard Galaxy model, as well as a few other luminosity functions obtained by varying the IMF slope at low mass. The luminosity function is made from segments of a power law IMF, as given in Eq. (1), and a mass luminosity relation from Delfosse et al. (2000) in the magnitude range $12<M_{V}<17$ and from theoretical models of Baraffe et al. (1998) at lower masses. The cutoff between absolute magnitude 16 to 18 is mostly due to the mass-luminosity relation and only weakly dependent on the IMF slope at the very low mass end. However in the range $13<M_{V}<16$ the luminosity function strongly depends on the assumed IMF slope and on the mass at which the slope changes:

$\frac{\mathrm{d} n}{\mathrm{~d} m} \propto m^{-\alpha}$

with:

$\alpha=1.5$ for $m<0.5 M_{\odot}$ (standard Galaxy model);

$\alpha=1.5$ for $m<0.5 M_{\odot}$ and $\alpha=2$ for $m<m_{\mathrm{c}}$; 


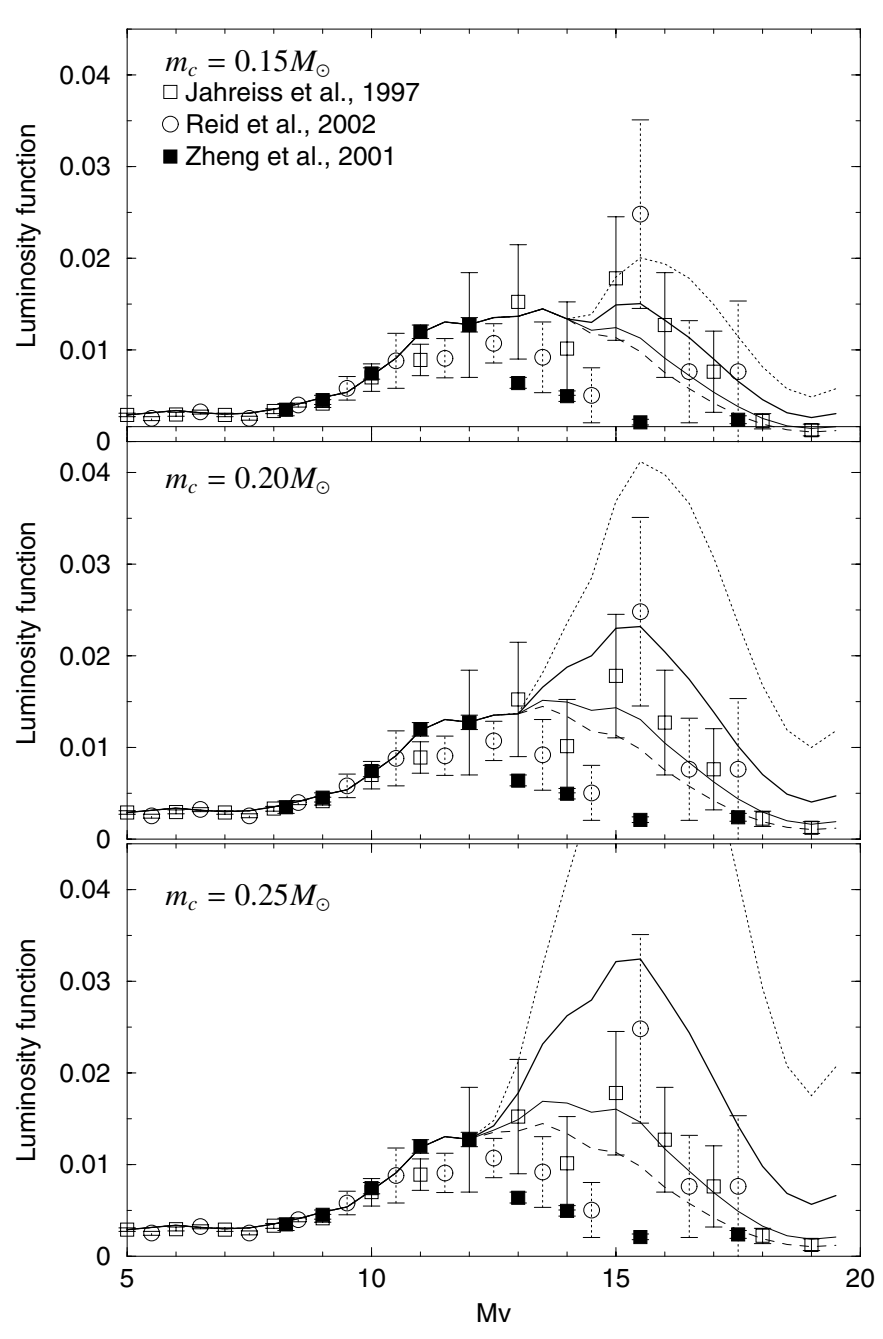

Fig. 11. Luminosity function in the $V$ band in number of stars per $\mathrm{pc}^{3}$ per magnitude for different break points in mass. The top panel indicates $m_{\mathrm{c}}=0.15 M_{\odot}$, the middle panel $m_{\mathrm{c}}=0.20 M_{\odot}$ and the lower panel $m_{\mathrm{c}}=0.25 M_{\odot}$. Open squares are from Jahreiss et al.'s (1997) determination from the revised Catalogue of Nearby Stars, open circles are from Reid et al. (2002) determination using the PMSU survey combined with Hipparcos data, filled squares are from Zheng et al.'s (2001) determination from the HST. The lines show model luminosity functions assuming different slopes for the IMF: $\alpha=1.5$ is the standard Galaxy model (dashed line), $\alpha=2$ (thin line), $\alpha=3$ (thick line), and $\alpha=2$ (dotted line) (see text).

$$
\begin{aligned}
& \alpha=1.5 \text { for } m<0.5 \quad M_{\odot} \text { and } \alpha=3 \text { for } m<m_{\mathrm{c}} \\
& \alpha=1.5 \text { for } m<0.5 \quad M_{\odot} \text { and } \alpha=4 \text { for } m<m_{\mathrm{c}}
\end{aligned}
$$

and $m_{\mathrm{c}}=0.15,0.20,0.25 M_{\odot}$.

In the following the various tested IMFs are denoted $\mathrm{LF} \alpha-m_{\mathrm{c}}$, where alpha is the IMF slope, and $m_{\mathrm{c}}$ is the mass where the slope changes.

We note that simulated stars considered here are single stars. Distant binary systems may be not correctly identified as stars, although from a detailed analysis of the binary effect (a complete analysis of the binary effect is postponed to the next paper of this series), we have estimated that at $r^{\prime}-i^{\prime}>1.6$ (that is $M_{V}>14$ ) the correction for stars missed for this reason is negligible. At $r^{\prime}-i^{\prime}<1.5$ the correction would be less
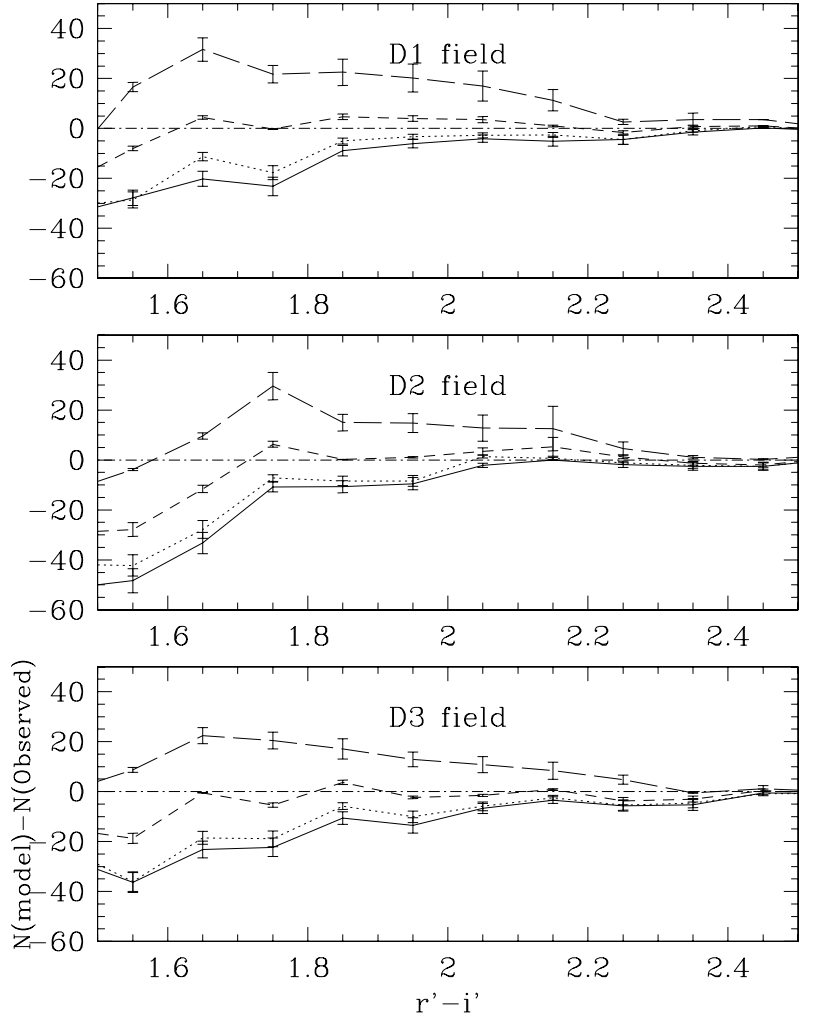

Fig. 12. Difference between model and data as a function of the $r^{\prime}-i$ colour for the D1, D2 and D3 field. The solid line indicates the standard model $(\alpha=1.5)$ with $m_{\mathrm{c}}=0.20 M_{\odot}$, the dotted line assuming $\alpha=2$, the short dashed and the long dashed line assuming $\alpha=3$ and $\alpha=4$ respectively, as explained in the text. The error bars are calculated assuming that the noise is due to the Poisson statistics.

than $25 \%$ and at $1.5<r^{\prime}-i^{\prime}<1.6$ it is less than $13 \%$. Hence with respect to the very faint end of the LF studied here, the binary effect is expected to be negligible because these stars are too close to have been missed even in binary systems. We limit our further comparison to $r^{\prime}-i^{\prime}>1.5$.

Figure 10 shows clearly a deficit of late type thin disc dwarfs in model predictions at $r^{\prime}-i^{\prime}>1.6$, for the standard Galaxy model. We have attempted to fit the LF to the available CFHTLS data in the 3 fields using the IMF formula from Eq. (1). Figure 12 shows the difference of the predicted star counts compared to observations for the three tested IMFs: $\alpha=2, \alpha=3$ and $\alpha=4$ with $m_{\mathrm{c}}=0.20 M_{\odot}$.

In Tables 4-6 we give the number of stars in $r^{\prime}-i^{\prime}$ intervals from the D1, D2 and D3 fields respectively, and, for comparison, the numbers simulated from tested LFs, varying $m_{\mathrm{c}}$ from 0.15 to $0.25 M_{\odot}$ and $\alpha$ at $m<m_{\mathrm{c}}$ from 1.5 to 4 . The standard deviation of the models relative to the data are estimated from Poisson statistics: assuming that the main source of error in the data is Poisson noise, we estimate the deviation of the model by computing:

$\sigma_{\text {model }}=\frac{N_{\text {data }}-N_{\text {model }}}{\sqrt{N_{\text {data }}}}$

which gives the relative difference in the counts in units of the Poissonian scatter. Acceptable models have $\sigma_{\text {model }}$ in the range $[-3,3]$. 
Table 4. Number of stars in the D1 field for $i^{\prime}<21$ and in different intervals of $r^{\prime}-i^{\prime}$. Column 1 indicates the middle of the interval of width 0.2 , "tot" means the total of the 3 interval considered, that is $1.5<r^{\prime}-i^{\prime}<2.1$. Columns 2 to 17 give the model counts for each of the tested LF while column 18 contains the observed counts. Models are described by two parameters: on the first line of the column heading the value of the mass of changing slope is indicated, the second line gives the value of $\alpha$ (see Eq. (1)). The column values include, in the first line, the number of stars in the colour interval, the second line gives the difference between the data and simulated counts in number of sigmas, assuming that the noise is dominated by the Poisson statistics (see text). Model with values between -3 and +3 are considered as acceptable in the colour bin.

\begin{tabular}{c|c|ccccc|cccccc|ccccc}
\hline \hline$r^{\prime}-i^{\prime}$ & std & .15 & .15 & .15 & .15 & .15 & .20 & .20 & .20 & .20 & .20 & .25 & .25 & .25 & .25 & .25 & data \\
& & 2 & 2.5 & 3 & 3.5 & 4 & 2 & 2.5 & 3 & 3.5 & 4 & 2 & 2.5 & 3 & 3.5 & 4 & \\
\hline 1.6 & 79 & 82 & 84 & 87 & 88 & 88 & 88 & 106 & 124 & 148 & 176 & 100 & 134 & 173 & 229 & 299 & 126 \\
$\sigma$ & 4.2 & 3.9 & 3.7 & 3.5 & 3.4 & 3.4 & 3.4 & 1.8 & 0.2 & -2.0 & -4.5 & 2.3 & -0.7 & -4.2 & -9.2 & -15.4 & \\
\hline 1.8 & 25 & 30 & 35 & 38 & 41 & 50 & 35 & 47 & 62 & 76 & 102 & 41 & 58 & 84 & 121 & 172 & 57 \\
$\sigma$ & 4.2 & 3.6 & 2.9 & 2.5 & 2.1 & 0.9 & 2.9 & 1.3 & -0.7 & -2.5 & -6.0 & 2.1 & -0.1 & -3.6 & -8.5 & -15.2 & \\
\hline 2.0 & 11 & 13 & 16 & 18 & 23 & 26 & 15 & 18 & 29 & 42 & 59 & 15 & 24 & 37 & 61 & 95 & 21 \\
$\sigma$ & 2.2 & 1.7 & 1.1 & 0.7 & -0.4 & -1.1 & 1.3 & 0.7 & -1.7 & -4.6 & -8.3 & 1.3 & -0.7 & -3.5 & -8.7 & -16.1 & \\
\hline tot & 115 & 125 & 135 & 142 & 151 & 165 & 138 & 171 & 215 & 266 & 337 & 157 & 216 & 294 & 411 & 566 & 204 \\
$\sigma$ & 6.2 & 5.5 & 4.8 & 4.3 & 3.7 & 2.7 & 4.6 & 2.3 & -0.8 & -4.3 & -9.3 & 3.3 & -0.8 & -6.3 & -14.5 & -25.3 & \\
\hline
\end{tabular}

Table 5. Same as Table 4 but for field D2.

\begin{tabular}{c|c|ccccc|ccccccc|ccccc}
\hline \hline$r^{\prime}-i^{\prime}$ & std & .15 & .15 & .15 & .15 & .15 & .20 & .20 & .20 & .20 & .20 & .25 & .25 & .25 & .25 & .25 & data \\
& & 2 & 2.5 & 3 & 3.5 & 4 & 2 & 2.5 & 3 & 3.5 & 4 & 2 & 2.5 & 3 & 3.5 & 4 & \\
\hline 1.6 & 80 & 75 & 81 & 81 & 87 & 89 & 91 & 106 & 122 & 146 & 167 & 101 & 134 & 176 & 235 & 310 & 141 \\
$\sigma$ & 5.1 & 5.6 & 5.1 & 5.1 & 4.5 & 4.4 & 4.2 & 2.9 & 1.6 & -0.4 & -2.2 & 3.4 & 0.6 & -2.9 & -7.9 & -14.2 & \\
\hline 1.8 & 28 & 29 & 32 & 35 & 39 & 48 & 33 & 40 & 56 & 70 & 94 & 36 & 53 & 76 & 112 & 156 & 49 \\
$\sigma$ & 3.0 & 2.9 & 2.4 & 2.0 & 1.4 & 0.1 & 2.3 & 1.3 & -1.0 & -3.0 & -6.4 & 1.9 & -0.6 & -3.9 & -9.0 & -15.3 & \\
\hline 2.0 & 9 & 10 & 14 & 17 & 20 & 24 & 14 & 18 & 26 & 36 & 49 & 13 & 23 & 33 & 55 & 85 & 21 \\
$\sigma$ & 2.6 & 2.4 & 1.5 & 0.9 & 0.2 & -0.7 & 1.5 & 0.7 & -1.1 & -3.3 & -6.1 & 1.7 & -0.4 & -2.6 & -7.4 & -14.0 & \\
\hline tot & 116 & 115 & 126 & 132 & 146 & 160 & 138 & 164 & 203 & 253 & 309 & 150 & 211 & 285 & 402 & 552 & 221 \\
$\sigma$ & 7.1 & 7.1 & 6.4 & 6.0 & 5.0 & 4.1 & 5.6 & 3.8 & 1.2 & -2.2 & -5.9 & 4.8 & 0.7 & -4.3 & -12.2 & -22.3 & \\
\hline
\end{tabular}

Table 6. Same as Table 4 but for field D3.

\begin{tabular}{c|c|ccccc|cccccc|cccccc}
\hline \hline$r^{\prime}-i^{\prime}$ & std & .15 & .15 & .15 & .15 & .15 & .20 & .20 & .20 & .20 & .20 & .25 & .25 & .25 & .25 & .25 & data \\
& & 2 & 2.5 & 3 & 3.5 & 4 & 2 & 2.5 & 3 & 3.5 & 4 & 2 & 2.5 & 3 & 3.5 & 4 & \\
\hline 1.6 & 75 & 71 & 76 & 76 & 78 & 81 & 81 & 100 & 116 & 141 & 167 & 93 & 125 & 160 & 208 & 288 & 126 \\
$\sigma$ & 4.5 & 4.9 & 4.5 & 4.5 & 4.3 & 4.0 & 4.0 & 2.3 & 0.9 & -1.3 & -3.7 & 2.9 & 0.1 & -3.0 & -7.3 & -14.4 & \\
\hline 1.8 & 22 & 25 & 31 & 34 & 39 & 44 & 31 & 40 & 54 & 69 & 93 & 36 & 51 & 75 & 111 & 161 & 57 \\
$\sigma$ & 4.6 & 4.2 & 3.4 & 3.0 & 2.4 & 1.7 & 3.4 & 2.3 & 0.4 & -1.6 & -4.8 & 2.8 & 0.8 & -2.4 & -7.2 & -13.8 & \\
\hline 2.0 & 10 & 12 & 15 & 18 & 22 & 26 & 14 & 18 & 26 & 36 & 54 & 15 & 24 & 39 & 60 & 96 & 30 \\
$\sigma$ & 3.7 & 3.3 & 2.7 & 2.2 & 1.5 & 0.7 & 2.9 & 2.2 & 0.7 & -1.1 & -4.4 & 2.7 & 1.1 & -1.6 & -5.5 & -12.0 & \\
\hline tot & 107 & 108 & 122 & 128 & 138 & 151 & 125 & 158 & 196 & 246 & 315 & 142 & 200 & 274 & 379 & 545 & 219 \\
$\sigma$ & 7.6 & 7.5 & 6.6 & 6.1 & 5.5 & 4.6 & 6.4 & 4.1 & 1.6 & -1.8 & -6.5 & 5.2 & 1.3 & -3.7 & -10.8 & -22.0 & \\
\hline
\end{tabular}

From these tables we can conclude that:

- The standard LF (with $\alpha=1.5$, i.e. no change of slope) is rejected at more than 6 sigmas in the three fields.

- The LF with a $m_{\mathrm{c}}=0.15 M_{\odot}$ is deficient in stars compared with the data in all three fields, regardless of the slope.

- Consistency with the data can be obtained with $m_{\mathrm{c}}=0.25$ or $m_{\mathrm{c}}=0.20 M_{\odot}$. Depending on which $m_{\mathrm{c}}$ we take, the value of $\alpha$ is slightly changed. Having a higher $m_{\mathrm{c}}$ allows a smaller $\alpha$. Constraints from the local LF (see Fig. 11) also indicate that having $m_{\mathrm{c}}>0.25 M_{\odot}$ would not allow large values for $\alpha$.

- The counts in all 3 fields are best fitted by an IMF slope of $\alpha=3$ (with $m_{\mathrm{c}}=0.20 M_{\odot}$ ) or $\alpha=2.5$ (with $\left.m_{\mathrm{c}}=0.25 M_{\odot}\right)$. As seen from Fig. 11 this LF is in acceptable agreement with the local LF as determined from the
25 pc sample. Comparing this IMF (either with $m_{\mathrm{c}}=0.20$ or $0.25 M_{\odot}$ ) with Chabrier (2003) log-normal LF, yields good agreement but the revised IMF from Chabrier (2004) gives a too few low mass stars for $0.10-0.15 M_{\odot}$ compared with our determination and with the local IMF. We emphasize that we have confirmed, from our intermediate distance sample that the disc IMF does not decrease for masses above the $0.1 M_{\odot}$ limit.

It should be noted that the number of stars decreases rapidly with the increasing $r^{\prime}-i^{\prime}$ colour. A slight change in the zero point of the photometric calibration in $r^{\prime}$ or $i^{\prime}$ may change slightly our conclusions. We estimate from Tables 4-6 that a systematic shift of 0.05 magnitude in $r^{\prime}-i^{\prime}$ would produce a change of about 0.5 on the slope $\alpha$. However such a shift is 
improbable as it would be seen also at the blue end of the sequence $\left(r^{\prime}-i^{\prime}=0\right)$ which is not the case, as seen in Fig. 9.

\subsection{Comparison with HST results}

Zheng et al. (2001) determined the luminosity function from a sample of about $1400 \mathrm{M}$ dwarfs in 148 fields using the WFC2 and 162 fields from PC1 with the HST. Their sample is characterized by a mean height above the plane of $1.5 \mathrm{kpc}$, with very few stars at vertical height $<1 \mathrm{kpc}$. They derive their LF and IMF taking into account a probable metallicity gradient, by adopting a metallicity of $-0.5 \mathrm{dex}$ at $1.5 \mathrm{kpc}$, and a colourabsolute magnitude relation varying with metallicity. Hence their sample is dominated by what we usually call the thick disc population. They deduce an IMF slope of $\alpha=-0.10$ or $\alpha=-0.47$ with or without the metallicity gradient taken into account.

The sample considered in this paper is significantly different from the HST sample, as it is dominated by stars at distances above the plane of 150 to $450 \mathrm{pc}$ with a mean distance of $350 \mathrm{pc}$ for stars at $r^{\prime}-i^{\prime}=1.6$ and $210 \mathrm{pc}$ for stars having $r^{\prime}-i^{\prime}=2.0$. This has two consequences: 1) the sample is less biased by unresolved binaries and 2) it is dominated by the normal thin disc population and more comparable with the local sample which is used to determine the LF in the solar neighbourhood (Reid et al. 2002).

Reylé \& Robin (2001) have performed the first determination of the thick disc IMF from a multi-directional analysis of star counts. They obtained an IMF $\mathrm{d} N / \mathrm{d} m \propto m^{-0.5}$ in the mass range $0.2<m<0.8 M_{\odot}$, which is in agreement with the IMF deduced by Zheng et al. (2001) from the HST sample, reinforcing the ideas that: firstly Zheng et al. (2001) have measured the thick disc IMF, rather than the thin disc one; secondly the thin disc and thick disc have different IMF slopes at low masses. The IMF found by Reylé \& Robin (2001) in the thick disc is well in agreement with the one determined in globular clusters (Paresece \& De Marchi 2000) and significantly different from the one found in the local thin disc (Kroupa 2001).

The origin of the thick disc has long been a matter of debate. Nowadays favoured scenarii explain the thick disc by one or more accretions of galaxy satellites at early epochs of the Galaxy's formation, or by star formation from gas accreted during a chaotic period of hierarchical clustering (Brook et al. 2004). The thick disc is old and metal poor relative to the sun and it is also enhanced in alpha elements. Abundance determinations (Gratton et al. 2000) also show that there has been a discontinuity in the star formation between the thick disc and the thin disc of at least 1 Gyr. The conditions of star formation at the epoch of thick disc formation were clearly different from the present conditions in the thin disc. Larson (2005) has analysed the physical conditions required for the thermal coupling of gas and dust in cloud fragmentation. He studied the roles of the metallicity, background radiation and dust environment on the Jeans mass, hence on the typical mass of the stars formed. The combined effects of the metallicity and the possible lack of dust at cosmological epochs could increase the peak mass of the IMF relative to the present one. Moreover as the cosmic background temperature was higher in the past, a higher minimum cloud temperature exists, which also implies a higher Jeans mass. These conditions may well explain the fact that the IMF found in the thick disc has a typical mass higher than the thin disc, and is deficient in the very low mass stars which are found in the present disk MF.

\section{Conclusions}

We have presented an analysis of the stellar populations in the CFHTLS using catalogues and images from the first public data release. Our population synthesis approach allowed us to test stellar libraries and to identify different stellar types and Galaxy components using colour-colour diagrams. We discuss the locations of various stellar species such as white dwarfs, late-type and brown dwarfs and binary systems in the MEGACAM filter/detector combination. The contamination of the stellar sample by quasars and compact galaxies is quantified using spectroscopic data from the VIMOS-VLT Deep Survey (VVDS). The percentage of the galaxy contamination depends very much on the $r^{\prime}-i^{\prime}$ colour and can reach a maximum of $23.5 \%$ for $i^{\prime}<22.0$ and $r^{\prime}-i^{\prime}<0.5$.

Our main conclusions concern the luminosity and mass function (MF) at low mass for the disc population. This data set favours an MF slope of $\alpha=2.5 \pm 1.0$ for $m<0.25 M_{\odot}$ or $\alpha=3.0 \pm 1.0$ for $m<0.2 M_{\odot}$ which although steep compared with previous investigations from other deep imaging surveys (such as Zheng et al. who used HST images) is still in agreement with local determinations of the IMF. This discrepancy can be explained by differences in the mean age and physical conditions of star formation of the samples, one being at about $1 \mathrm{kpc}$ or more where the thick disc population is expected to dominate, and ours being at $150-450 \mathrm{pc}$ and dominated by the thin disc. This discrepancy between the thin disc and thick disc IMFs could be explained if for physical reasons (for example, lack of dust, higher temperature backgound radiation or metallicity) very low mass star formation has been less efficient at the epoch of the thick disc formation.

The new IMF as determined here cannot be extrapolated to masses below $0.1 M_{\odot}$. It is probable from the numbers of known brown dwarfs in clusters that the IMF starts to decrease near the $\mathrm{H}$ burning limit (Kroupa 2001).

In future papers we plan a more detailed analysis of these stellar populations, in particular the IMF at low masses of the disk, thick disk and spheroid and the old population density distribution up to several tens of kiloparsecs.

This might be performed using more accurate star-galaxy separation and by accounting for binary frequency in the modelling. The combination of multiband wide survey coverage together with proper motions will enable us to count thick disc and halo white dwarfs, and to constrain on the fraction of baryonic dark matter present in the form of stellar remnants.

Acknowledgements. We would like to warmly thank Roser Pello for providing us with simulated galaxy colours in the MEGACAM photometric systems for a range of different redshifts, Pierre Bergeron for providing his white dwarf models and Thibault Lejeune for his invaluable assistance in the computation of the synthetic colours from the 
stellar libraries. We would like also to thank the VVDS consortium for providing us with spectroscopic observations. Many thanks also to Bernard Debray who is responsible for providing the web interface for the Besancon Galaxy model. MS is supported by an APART fellowship.

\section{References}

Allard, F., Hauschildt, P. H., Alexander, D. R., \& Starrfield, S. 1997, ARA\&A, 35, 137

Baraffe, I., Chabrier, G., Allard, F., \& Hauschildt, P. H. 1998, A\&A, 337,403

Bergeron, P., Wesemael, F., \& Beauchamp, A. 1995, PASP, 107, 1047

Bergeron, P., Leggett, S. K., \& Ruiz, M. T. 2001, ApJS, 133, 413

Bertin, E., \& Arnouts, S. 1996, A\&A, 117, 393

Blaizot, J., Guideroni, B., Devriendt, J. E. G., et al. 2004, MNRAS, 352,571

Brook, C. B., Kawata, D., Gibson, B. K., \& Freeman, K. C. 2004, ApJ, 612,894

Burgasser, A. J., McElwain, M. W., Kirkpatrick, J. D., et al. 2004, AJ, 127,2856

Buser, R., \& Kurucz, R. L. 1992, A\&A, 264, 557

Chabrier, G., \& Baraffe, I. 2000, ARA\&A, 38, 337

Chabrier, G. 2003, ApJ, 586, L133

Chabrier, G. 2004, Invited review "IMF@ 50" in honor of E. Salpeter, preprint [arXiv: astro-ph/0409465]

Crézé, M., Chereul, E., Bienaymé, O., \& Pichon, C. 1998, A\&A, 329, 920

Crézé, M., Mohan, V., Robin, A. C., et al. 2004, A\&A, 426, 65

Delfosse, X., Forveille, T., Ségransan, D., et al. 2000, A\&A, 364, 217

Fan, X., Knapp, G. R., Strauss, M. A., et al. 2000, AJ, 119, 928

Golimowski, D. A., Leggett, S. K., Marley, M. S., et al. 2004, AJ, 127 , 3516

Gelino, C. R., Kirkpatrick, J. D., \& Burgasser, A. J. 2004, American Astronomical Society Meeting Abstracts, 205

Gómez, A. E., Grenier, S., Udry, S., et al. 1997, ESA SP-402, Hipparcos, Venice '97, 621

Gratton, R. G., Carretta, E., Matteucci, F., \& Sneden, C. 2000, A\&A, 358,671

Hawley, S. L., Covey, K. R., Knapp, G. R., et al. 2002, AJ, 123, 3409

Haywood, M., Robin, A. C., \& Crézé, M. 1997a, A\&A, 320, 428
Haywood, M., Robin, A. C., \& Crézé, M. 1997b, A\&A, 320, 440

Henry, T. J., Ianna, P. A., Kirkpatrick, J. D., \& Jahreiss, H. 1997, AJ, 114,388

Holberg, J. B., Oswalt, T. D., \& Sion, E. M. 2002, ApJ, 571, 512

Jahreiß, H., \& Wielen, R. 1997, ESA SP-402, Hipparcos - Venice '97, 675

Kendall, T. R., Delfosse, X., Martín, E. L., \& Forveille, T. 2004, A\&A, 416, L17

Kroupa, P. 2001, MNRAS, 322, 231

Kurucz, R. L. 1979, ApJS, 40, 1

Larson, R. B. 2005, MNRAS, 359, 211

Lejeune, Th., Cuisinier, F., \& Buser, R. 1997, A\&AS, 125, 229

Le Fèvre, O., Vettolani, G., Garilli, B., et al. 2005, A\&A, 439, 845

Liebert, J., Dahn, C., \& Monet, D. 1988, ApJ, 332, 891

McCracken, H. J., Radovich, M., Bertin, E., et al. 2003, A\&A, 410, 17

Ojha, D. K., Bienaymé, O., Robin, A. C., Crézé, M., \& Mohan, V. 1996, A\&A, 311, 456

Ojha, D. K., Bienaymé, O., Mohan, V., \& Robin, A. C. 1999, A\&A, 351, 945

Paresce, F., \& De Marchi, G. 2000, ApJ, 534, 870

Picaud, S., \& Robin, A. C. 2004, A\&A, 428, 891

Raymond, S. N., Szkody, P., \& Hawley, S. L. 2003, AJ, 125, 2621

Reid, I. N., Gizis, J. E., \& Hawley, S. L. 2002, AJ, 124, 2721

Reylé, C., \& Robin, A. C. 2001, A\&A, 373, 886

Robin, A. C., Haywood, M., Crézé, M., Ojha, D. K., \& Bienaymé, O. 1996, A\&A, 305, 125

Robin, A. C., Reylé, C., \& Crézé, M. 2000, A\&A, 359, 103

Robin, A. C., Reylé, C., Derrière, S., \& Picaud, S. 2003, A\&A, 409, 523

Ruiz, M. T., \& Bergeron, P. 2001, ApJ, 558, 761

Schneider, D. P., Knapp, G. R., Hawley, S. L., et al. 2002, AJ, 123, 458

Schneider, D. P., Fan, X., \& Hall, P. B. 2003, AJ, 126, 2579

Strauss, M. A., Fan, X., Hall, P. B., et al. 1999, ApJ, 552, L61

Szalay, A. S., Jain, B., Matsubara, T., et al. 2003, ApJ, 591, 1

Tsvetanov, Z. I., Golimowski, D. A., Zheng, W., et al. 2000, ApJ, 531, L61

Westera, P., Lejeune, T., Buser, R., Cuisinier, F., \& Bruzual, G. 2002, A\&A, 381, 524

Zheng, Z., Flynn, C., Gould, A., Bahcall, J., \& Salim, S. 2001, ApJ, 555,393 